\title{
Comparison of a Chinese Herbal Medicine (CCH1) and Lactulose as First-Line Treatment of Constipation in Long-Term Care: A Randomized, Double-Blind, Double-Dummy, and Placebo-Controlled Trial
}

\author{
Chien-Hsun Huang, ${ }^{1,2}$ Jui-Shan Lin, ${ }^{3}$ Tsai-Chung Li, ${ }^{4}$ Shih-Chang Lee, ${ }^{3}$ \\ Hsiu Po Wang, ${ }^{5}$ Hung-Chi Lue, ${ }^{6}$ and Yi-Chang $\mathrm{Su}^{2,3}$ \\ ${ }^{1}$ Department of Community and Family Medicine, National Taiwan University Hospital Yun-Lin Branch, Yun-Lin, Taiwan \\ ${ }^{2}$ Graduate Institute of Chinese Medicine, College of Chinese Medicine, China Medical University, Taichung, Taiwan \\ ${ }^{3}$ School of Chinese Medicine, College of Chinese Medicine, China Medical University, Taichung, Taiwan \\ ${ }^{4}$ Graduate Institute of Biostatistics, China Medical University, Taichung, Taiwan \\ ${ }^{5}$ Division of Gastroenterology, Department of Internal Medicine, National Taiwan University Hospital, Taipei, Taiwan \\ ${ }^{6}$ Department of Pediatrics, National Taiwan University Hospital, Taipei, Taiwan \\ Correspondence should be addressed to Yi-Chang Su, juishan.lin@msa.hinet.net
}

Received 10 October 2011; Revised 21 November 2011; Accepted 22 November 2011

Academic Editor: Angelo Antonio Izzo

Copyright () 2012 Chien-Hsun Huang et al. This is an open access article distributed under the Creative Commons Attribution License, which permits unrestricted use, distribution, and reproduction in any medium, provided the original work is properly cited.

\begin{abstract}
Many institutionalized patients and their healthcare providers are dissatisfied with current laxative therapy. This study compared therapeutic efficacy, safety, and laxative cost of an herbal formula $(\mathrm{CCH} 1)$ and lactulose for long stay patients with constipation. In this double-blind, double-dummy, and placebo-controlled trial, we randomized 93 residents with chronic constipation from two long-term care facilities in Taiwan to receive either $\mathrm{CCH} 1$ with lactulose placebo or CCH1 placebo with lactulose for 8 weeks, then followed up for 4 weeks without study medication. Both treatments were effective and well tolerated for patients, but CCH1 produced more spontaneous bowel movements, less rectal treatments, less amount of rescue laxative, and lower laxative cost than lactulose during treatment. No significant differences were found in stool consistency, stool amount, global assessment, and safety concerns. In conclusion, our results suggest that $\mathrm{CCH} 1$ may have better efficacy and could be used as an alternative option to lactulose in the treatment of constipation in long-term care.
\end{abstract}

\section{Introduction}

Constipation is often dismissed as a trivial medical concern, it can be associated with mild-to-extreme distressing and potentially dangerous complications [1]. Almost half of all patients older than 65 reported constipation or the regular use of laxatives [2]. In nursing facilities, $72.7 \%$ of the patients had at least one precipitating comorbid condition for constipation, and $77.6 \%$ of the residents were receiving at least one medication that may contributed to the occurrence of constipation [3]. Accordingly, the prevalence of constipation is higher in long-term care with up to $74 \%$ of nursing home residents using daily laxatives [4]. In fact, constipation often leads to deterioration in health-related quality of life [5] and also increases time and economic cost of constipation care in long stay [6].

Dissatisfied with conventional therapy, there is a trend that herbal medicine use increased substantially in the United States, and over one in three Americans used complementary and alternative medicine (CAM) in the past year [7]. In Taiwan, more than $95 \%$ of citizens have medical insurance under the national health insurance (NHI) system. The use of Chinese medicine is also reimbursed by the NHI, and all qualified residents in Taiwan are free to choose either Western medicine or traditional Chinese medicine (TCM) [8]. A national survey in Taiwan reported 152,564 subjects 
who visited TCM clinics only for constipation during 2004 [9]. Constipation is also of the top three main diagnoses in a Traditional Medicine Center of a general hospital in Taiwan [10].

In fact, TCM has been the primary system of medicine in Asia for thousands of years. According to the theories of TCM, constipation can be generally divided into excessive and deficient syndromes (ying, yang, Qi or blood deficiency), based on the underlying etiology $[11,12]$. With regard to the syndrome of yang deficiency, it is a pathological state resulting from deficiency of "yang" energy with reduction in its warming and activating power that leads to diminished functions (including GI system), decreased metabolic activities, intolerance to cold, and so on $[13,14]$. "Wen-Pi Tang", derived from a classic TCM book named Qian Jin Fang (chapter 15) in the Tang Dynasty (A.D. 652), is a famous formula that has been commonly used throughout Asia for constipation of the "yang deficiency" type $[15,16]$. It was composed of five herbs (Ren Shen, Gan Jiang, Zhi Fu Zi, Da Huang, and Gan Cao) $[15,16]$. Through the combined action of these herbs, Da Huang served as purgative drug with the assistant of the rest of four herbs formulated to strengthen the energy of yang $[14,16]$. From the viewpoints of TCM, the majority of patients with constipation of yang deficiency are the frail older people or chronically ill patients $[11,15]$. Since many residents in nursing homes are the dependently older people with comorbidities, most of their constipation could be attributed to yang deficiency in some degree. Therefore, our previous randomized placebo-controlled trail has demonstrated CCH1 (Table 1), an herbal formula modified from "Wen-Pi Tang", to be safe and effective on the treatment of constipation for the population in nursing homes [17].

Despite the enthusiasm for CAM, the high-quality comparative study of herbal supplements and western medicine for constipation is limited $[18,19]$. From the review of literature, osmotic laxatives are effective in older adults and well tolerated $[1,20]$. in the UK, the most commonly used of osmotic agents is lactulose [21], which is also popularly used in Taiwan. therefore, we aimed to compare the efficacy, safety, and cost of $\mathrm{CCH} 1$ and lactulose for treating constipation in Long-Term Care.

\section{Materials and Methods}

2.1. Participants. The participants were the residents of two nursing homes in mid Taiwan. They received usual medical care from one surrounding teaching hospital in the same community. The study was conducted from August 2008 through April 2010. The inclusion and exclusion criteria are shown in Table 2.

The study was performed in compliance with the principles of the Declaration of Helsinki and following "good clinical practice" guidelines. The study protocol was approved by the Research Ethics Committee of National Taiwan University Hospital before conducted.

2.2. Study Medication. The herbal preparation was obtained from Sun Ten international pharmaceutical company,
TABLE 1: The composition of CCH1.*

\begin{tabular}{llc}
\hline $\begin{array}{l}\text { Manderin } \\
\text { pronunciation }\end{array}$ & Botanical name & Gram \\
\hline Ren Shen & Panax ginseng C. A. Meyer & 0.8 \\
Gan Jiang & Zingiber officinale Rosc. & 0.8 \\
Gan Cao & Glycyrrhiza uralensis Fisch. & 0.8 \\
Bai Zhu & Atractylodes macrocephala Koide. & 0.8 \\
Zhi Fu Zi & Aconitum carmichaeli Debx. & 0.8 \\
Da Huang & Rheum tanguticum Maxim. ex Balf. & 1.2 \\
\hline
\end{tabular}

*Every $3.0 \mathrm{~g}$ extract powder are prepared from the above raw herbs.

a qualified manufacturer of concentrated herbal extracts with good manufacturing practice. The herbs were authenticated at the Brion Research Institute in Taiwan on the basis of standards specified in the Taiwan Pharmacopoeia of Chinese Medicine (2004 edition). Contamination screening for heavy metals, pesticides, and aflatonix was performed to ensure safety for human consumption. For quality control and standardization of $\mathrm{CCH} 1$, herbal preparation was performed in a single batch to ensure consistency of quality. The indicator ingredient of CCH1 was analyzed by high-performance liquid chromatography (HPLC). After decoction and extraction, herbal preparation was concentrated into the powdered form and packed in sealed opaque aluminum foil bags.

With the reference of a double-blind, and placebocontrolled trial of an herbal formula [22], the placebo of $\mathrm{CCH} 1$ was made of similarly colored starch, which was packed in an identical package and manufactured by the same company as CCH1. With the other reference of a study for elderly constipated patients, $50 \%$ of glucose water was used as placebo-controlled of lactulose [23]. For similar viscosity and taste with lactulose, the placebo of lactulose in our study was manufactured by the same company as lactulose syrup, which was composed of $500 \mathrm{mg}$ glucose, $2 \mathrm{mg}$ xanthum gum (a polysaccharide used as a food additive to increase the viscosity of a liquid), $0.01 \mathrm{mg}$ Sunset yellow FCF (an artificial coloring allowed to be added to food), and $497.99 \mathrm{mg}$ purified water in each gram content. For the ingredients of placebo in this study, no known laxative effects or drug interaction with study medication existed.

2.3. Study Design. This double-blind, double-dummy, and placebo-controlled study had two parallel groups with a total treatment phase of 8 weeks. After 8 weeks of treatment, patients were followed up for one additional month without study medication (CCH1 or lactulose) and placebo except for rescue laxative of magnesium oxide ( $\mathrm{MgO})$. To obtain baseline information, all patients had a run-in period of 2 weeks without laxatives but only concurrent use of $\mathrm{CCH} 1$ placebo and lactulose placebo for ethical concerns and test for compliance before randomization.

With the reference of the national guidelines of the Registered Nurses Association [24], daily stool diaries were kept by the certified nurse assistants from the beginning of the run-in period to the completion of the study, including stool frequency, stool consistency, stool amount, and the use of rectal treatment (RT; enema, suppository use, or 
TABLE 2: Inclusion and exclusion criteria.

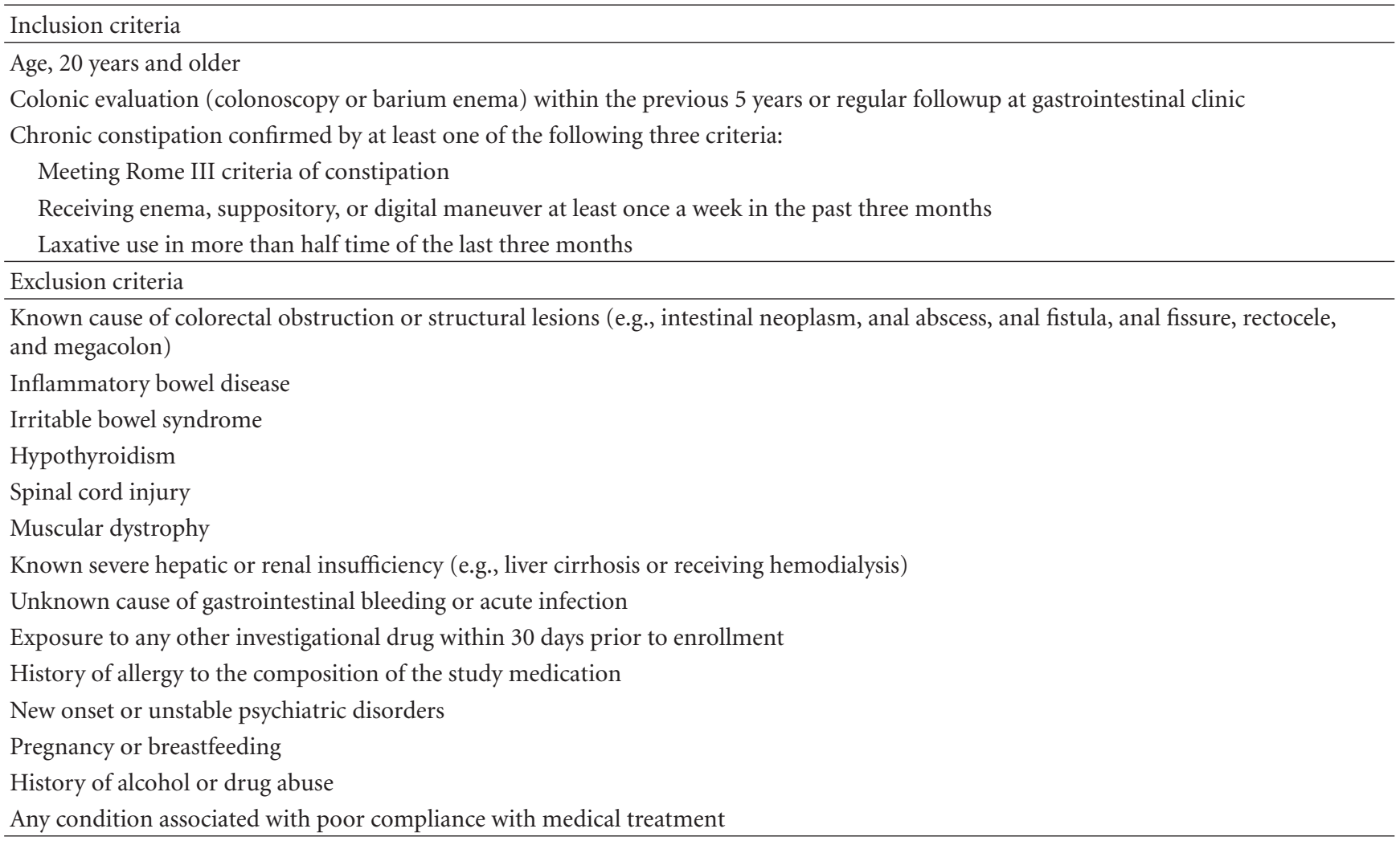

digital maneuver). Stool consistency was recorded by Bristol Stool Form Scale, ranging from 1 (separate hard lumps, like nuts) to 7 (watery, no solid pieces) [25]. Stool amount was classified into three categories (small, moderate, and large) according to the national guidelines [24]. In addition, a bowel routine protocol was set: if there was no bowel movement (BM) by day 3, the caregiver gave a suppository of bisacodyl or glycerin ball on day 3; if there was no BM after 8 hours of suppository insertion, enema was given. Meanwhile, when necessary, the caregiver could apply a digital maneuver to manually remove the hard stool or stimulate the rectum. Therefore, the bowel performance kept in the stool diary could imply the severity of constipation clinically.

Considering of the real situation in long-term care, physicians usually prescribe laxatives with different dosage according to the severity of constipation based on the stool diary. The participants were classified into three groups for initial dosage of study medication by enema frequency and weekly spontaneous bowel movement (SBM) during the run-in period. Having enema more than once a week was regarded as group A. Residents who received enema once a week or had less than three SBMs per week belonged to the group B. The frequency of SBM three to seven times a week was referred to as group C. Participants averaging more than one SBM per day during the run-in period were excluded.

Residents were randomized to the study medication according to a computer-generated randomization schedule. Randomization was stratified based on the severity of constipation and Barthel's index [26], a scale used to measure functional performance in basic activities of daily living. Allocation concealment was performed by enclosing assignments in sequentially numbered, identical, and sealed two envelopes according to the allocation sequence in every stratified group. The packaging of study medication and placebo were indistinguishable, and they were dispensed after randomization by an independent research assistant in a separate office. All persons involved in the conduct and management of the study were blinded to individual patient treatment during the study.

2.4. Interventions. The experimental group received an initial dose of 4.5/3.0/1.5 gm CCH1 powder with 45/30/15 mL lactulose placebo per day for groups $\mathrm{A} / \mathrm{B} / \mathrm{C}$, respectively. The active comparator group was given an initial dose of 45/30/15 mL lactulose with 4.5/3.0/1.5 gm CCH1 placebo per day for groups $\mathrm{A} / \mathrm{B} / \mathrm{C}$, respectively. If optimal bowel performance [27] (3SBMs/wk to $3 \mathrm{SBM}$ /day without any rectal treatment) was not reached, study medication were titrated by weekly increments of " $1.5 \mathrm{gm}$ of CCH1 and $15 \mathrm{~mL}$ of lactulose placebo for experimental group" or " $15 \mathrm{~mL}$ of lactulose and $1.5 \mathrm{gm}$ of $\mathrm{CCH} 1$ placebo for comparator group" until the maximal daily dose of $6.0 \mathrm{gm}$ for $\mathrm{CCH} 1 /$ CCH1 placebo or $60 \mathrm{~mL}$ for lactulose/lactulose placebo. Daily dosage over 3.0 gm of $\mathrm{CCH} 1 / \mathrm{CCH} 1$ placebo or over $30 \mathrm{~mL}$ of lactulose/lactulose placebo was prescribed by divided dose.

During the whole study period of 12 weeks, $\mathrm{MgO}$ was the only rescue oral laxative allowed to be used. In the treatment 
phase of weeks $1-8$, if optimal bowel performance was not reached under the maximal dose of study medication, $\mathrm{MgO}$ was added by the blinded primary physician and was titrated every week (increased by up to $750 \mathrm{mg}$ /day once a week, maximum dose of $2.0 \mathrm{~g} /$ day).

2.5. Assessment. Participants were visited every week by one primary physician with licenses for both conventional medicine and TCM during the entire study period of 12 weeks. SBM was defined as stool passage without digital maneuver and without the use of suppository or enema on the same day. The cost calculations of lactulose and $\mathrm{MgO}$ were derived from the reimbursed payment of the national health insurance in Taiwan. The cost assigned to the investigational product $(\mathrm{CCH} 1)$ was derived from current wholesale distributor pricing. Using a 5-point Likert scale, the global assessment of efficacy was evaluated by patients themselves or their principal caregivers if their cognition was impaired.

2.6. Efficacy. The frequency of SBM during weeks 1-4, 58, and 9-12 was measured as primary efficacy. Secondary end points included (1) frequency of RT during weeks 1$4,5-8$, and $9-12$; (2) weekly amount of rescue laxative use during weeks 1-4, 5-8, and 9-12; (3) weeky laxative cost during treatment phase of weeks 1-4 and 5-8; (4) average score of Bristol Scale during weeks 1-4, 5-8, and 9-12; (5) mean proportion of different stool amount (small, moderate, and large) per week during weeks $1-4,5-8$, and 9-12; (6) global assessment of efficacy at the end of treatment phase; (7) cumulative incidence of adverse events during the entire study period.

2.7. Safety. Safety evaluations included adverse events and serious adverse events reporting, vital signs, physical examination findings, and laboratory results. Adverse events were monitored with a comprehensive symptom questionnaire as well as clinical laboratory testing-including complete blood count, plasma sugar, aspartate aminotransferase, alanine aminotransferase, total bilirubin, alkaline phosphatase, urea nitrogen, creatinine, albumin, thyroid-stimulating hormone, free thyroxine, sodium, potassium, calcium, phosphate, magnesium, uric acid, triglyceride, and total cholesterol—carried out before and after treatments in a qualified laboratory.

2.8. Statistical Analysis. An intention to treat analysis was conducted on all patients randomized for therapy. All patients who had taken at least one dose of study medication after randomization were included in the safety analysis. A last observation carried forward analysis was conducted for any missing data of primary or secondary outcomes except global assessment of efficacy and adverse events. Since primary efficacy and some secondary end points were to measure the outcomes at different time periods, the Bonferroni adjustment for multiple comparisons was used to assess the statistical significance of these multiple tests. Statistical analysis was performed using SPSS software version 13 (SPSS, Chicago, IL, USA). Numerical data were compared using the Student's $t$-test or Mann-Whitney $U$ test. The paired $t$-test was used to compare individual bowel performance before and after treatment. A $\chi^{2}$ test or Fisher exact test where appropriate were used to compare the number of patients with adverse events, global assessment of efficacy, and categorical data of patients' characteristics in baseline period between two groups. These comparisons were made at a 2 -sided $\alpha$ level of 0.05 .

2.9. Sample Size Considerations. Based on previous clinical trial of $\mathrm{CCH} 1$ and the methodological recommendations of a systematic review $[17,21]$, the standard deviation of the mean weekly SBM was estimated to be 2.0. A total sample size of about 93 patients would be required to detect a mean difference between treatments of 1.5 bowel movements per week under the assumptions of $90 \%$ power, a 2 -sided $\alpha$ value of 0.05 and allowance for a $20 \%$ drop-out rate.

\section{Results}

3.1. Participants, Study Conduct, and Completion. A total of 120 participants who had signed the informed consent were screened for eligibility; 93 (77.5\%) met the inclusion criteria and were randomized. The reasons for exclusion are shown in Figure 1. Over $80 \%$ of the 93 enrolled patients were older than 60 years with mean age of 73.5 and $54.8 \%$ were female. Patients' baseline characteristics were similar between the two treatment groups (all $P<0.05$; Table 3 ). A total of 20 $(21.5 \%)$ patients dropped out of the trial during the treatment phase (weeks 1-8): 5 (11.4\%) in the CCH1 group and $15(30.6 \%)$ in the lactulose group $(P=0.041)$. Among the 15 dropouts in lactulose group, 8 people had severe adverse events and hospitalized (pneumonia: 2, acute gastroenteritis: 2, upper gastrointestinal bleeding: 1 , urinary tract infection: 1, ileus: 1 , and cellulitis: 1); 2 persons withdrew their consents and one of them complained lactulose syrup was too sweet; 2 residents were lost to followup because transferred to other facilities; one person was withdrawn on the ethical concerns for refractory constipation under the maximum dosage of treatment medication and rescue laxative; another 2 persons were dropped from the study for poor compliance (Figure 1). Thirty-four (77.3\%) patients in the $\mathrm{CCH} 1$ group and $29(59.2 \%)$ patients in the lactulose group completed the study $(P=0.077)$. There was no significant different on demographics between drop outs and nondrop outs.

3.2. Primary Efficacy Analysis. During treatment phase, mean daily dose of study medication was 3.8 (standard deviation 1.4) gram CCH1 in experimental group and 38 (SD 14) $\mathrm{mL}$ lactulose in comparator group. No significant differences were found in the frequency of SBM in baseline period between the two groups (mean difference 0.1 [95\% CI -0.9 to 1.0]). Mean numbers of weekly SBM in CCH1 group were greater than those in lactulose group during 8-week treatment phase (6.8 versus 5.0, difference 1.8 [0.7 to 3.0]; $P=$ $0.001)$; greatest difference was during weeks $1-4$ (6.9 versus 4.5, difference 2.4 [1.2 to 3.6]; $P<0.001$ ). However, in the follow-up phase of weeks 9-12, the frequency of SBM in $\mathrm{CCH} 1$ group was less than that in lactulose group (3.7 versus 


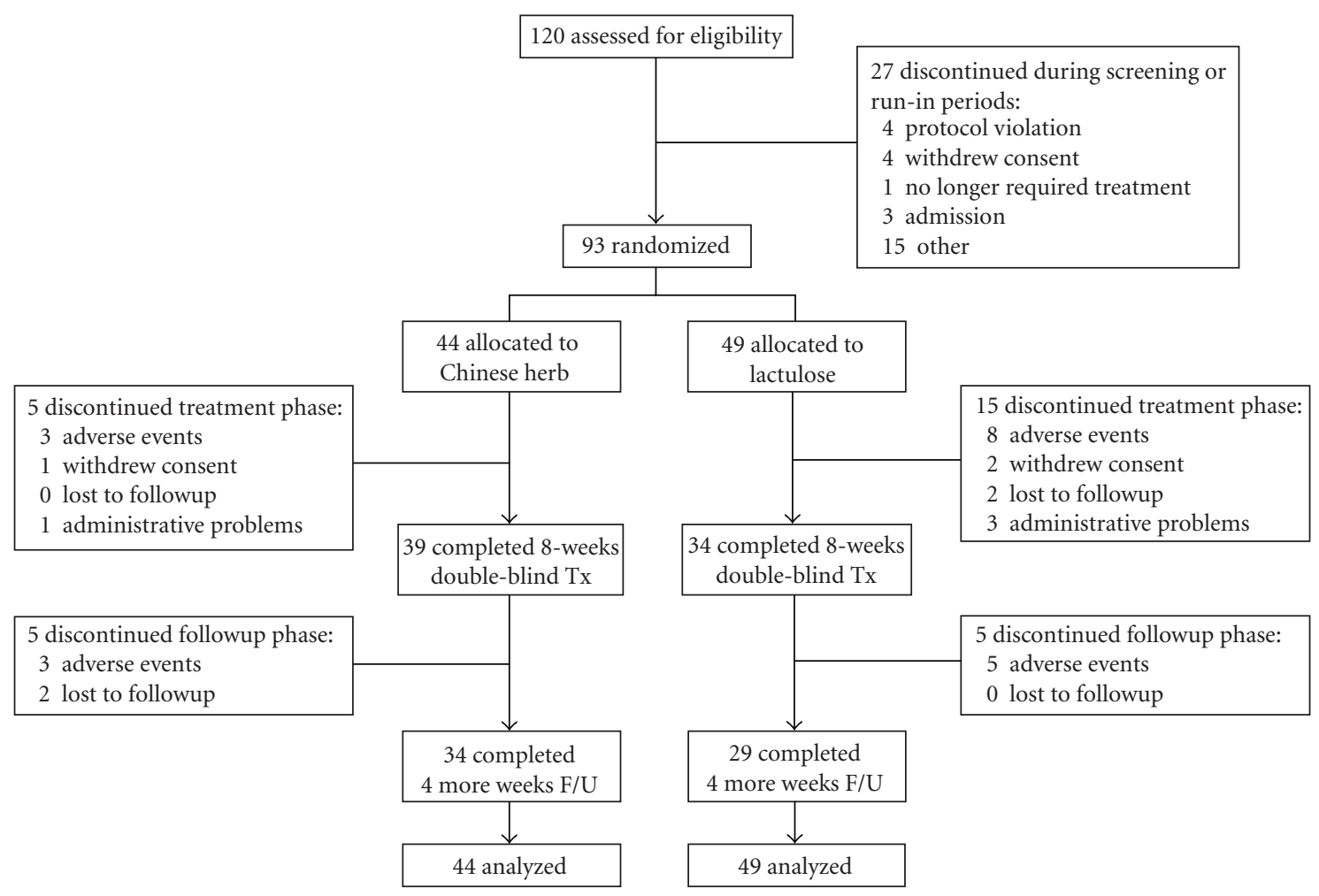

FIGURE 1: Participant flowchart depicting the randomization, treatment, and followup in CCH1 and lactulose groups.

5.2; $P=0.084)$,but still greater than its baseline period $(3.7$ versus 2.7; $P=0.005$ ) (Table 4 ).

3.3. Secondary End Points. Smaller mean numbers of RT/wk were observed in the CCH1 group compared with the lactulose group during weeks $1-4$ ( 0.5 versus 0.9 , difference $-0.4[-0.7$ to -0.1$])$ and weeks $5-8(0.3$ versus 0.6 , difference $-0.3[-0.5$ to -0.1$])$. Regarding the need for a rescue laxative $(\mathrm{MgO})$, the mean numbers of tablets of $\mathrm{MgO} / \mathrm{wk}$ were less for the patients who were given $\mathrm{CCH} 1$ than for those who received lactulose during the entire treatment phase; the greatest difference was during weeks 5-8 (1.7 versus 10.4 , difference $-8,7$ [ -13.4 to -3.9$]$ ). However, no significant differences were found in the frequency of RT/wk ( 1.0 versus 0.8 ) or in the amount of $\mathrm{MgO} / \mathrm{wk}$ (19.1 versus 20.0) between the two groups during follow-up phase of weeks 9-12 (Table 4).

Compared with the lactulose group, the laxative cost (USD/wk) in CCH1 group was less during weeks 1-4 (1.4 versus 4.7 , difference $-3.3[-3.8$ to -2.8$]$ ) and weeks $5-8$ (1.5 versus 5.0, difference $-3.5[-4.2$ to -2.8$]$ ) (Table 4$)$. No significant differences were found in stool consistency or stool amount between the two groups at all time points (Table 5). The $73(78.5 \%)$ patients who completed the treatment phase were investigated for global assessment of efficacy at week $8,84.6 \%$ of $\mathrm{CCH} 1$ patients had marked or slightly improvement versus $79.4 \%$ in the lactulos group $(P=0.646)$ (Table 6).
3.4. Safety and Compliance. All patients in both groups had good compliance of around $97 \%$ with study medication except for three patients with 70-80\% (Table 7). Six (13.6\%) in $\mathrm{CCH} 1$ and $12(24.5 \%)$ in lactulose patients discontinued the study because of severe adverse events (SAEs) $(P=0.203$; Table 8 ). No significant differences were found between the two groups in the incidence of any one of the common AEs or SAEs (Tables 8 and 9).

\section{Discussion}

In this randomized, double-blind, double-dummy, and placebo-controlled trial, we found that both $\mathrm{CCH} 1$ and lactulose are effective and well tolerated in long stay patients with constipation. However, the regimen of $\mathrm{CCH} 1$ produced more frequent SBM, less frequent RT, lower amount of rescue laxative, and lower laxative cost than lactulose during treatment of 8 weeks.

In TCM, "syndromes" are the foundations for therapeutic principles [11]. However, to date there is no international consensus or consistently applied classification or criteria of syndromes for many diseases and symptoms such as constipation [28]. And the process of syndrome identification performed by TCM doctors is very subjective and usually lacks interrater consistency because it is highly dependent on the TCM doctors' personal skills including evaluating the nature of patient's pulse and appearance of the tongue [28]. That is why clinical trials of TCM are often questionable 
TABLE 3: Characteristics of patients in baseline period.

\begin{tabular}{|c|c|c|c|}
\hline \multirow{2}{*}{ Variables } & \multicolumn{2}{|c|}{ Group, number (\%) } & \multirow{2}{*}{$P$ value } \\
\hline & $\mathrm{CCH} 1$ & Lactulose & \\
\hline \multicolumn{4}{|l|}{ Gender } \\
\hline Female & $26(59.1)$ & $25(51.0)$ & \multirow{2}{*}{0.43} \\
\hline Male & $18(40.9)$ & $24(49.0)$ & \\
\hline \multicolumn{4}{|l|}{ Age (years) } \\
\hline $41-60$ & $9(20.5)$ & $9(18.4)$ & \multirow{3}{*}{0.59} \\
\hline $61-80$ & $17(38.6)$ & $24(49.0)$ & \\
\hline$>80$ & $18(40.9)$ & $16(32.7)$ & \\
\hline Mean (SD) & $73.4(13.3)$ & $73.6(13.2)$ & 0.94 \\
\hline \multicolumn{4}{|l|}{ Abdominal surgery } \\
\hline No & $30(68.2)$ & $38(77.6)$ & \multirow{2}{*}{0.30} \\
\hline Yes & $14(31.8)$ & $11(22.4)$ & \\
\hline \multicolumn{4}{|l|}{ Barthel index* } \\
\hline$\leq 30$ & $31(70.5)$ & $37(75.5)$ & \multirow{3}{*}{0.83} \\
\hline $35-60$ & $6(13.6)$ & $5(10.2)$ & \\
\hline$>60$ & $7(15.9)$ & $7(14.3)$ & \\
\hline Median (25th, 75th percentile) & $17.5(0,45)$ & $10.0(0,32.5)$ & 0.59 \\
\hline \multicolumn{4}{|l|}{ Severity of constipation ${ }^{\dagger}$} \\
\hline Group A & $21(47.7)$ & $23(46.9)$ & \multirow{3}{*}{0.84} \\
\hline Group B & $15(34.1)$ & $19(38.8)$ & \\
\hline Group C & $8(18.2)$ & $7(14.3)$ & \\
\hline \multicolumn{4}{|l|}{ History of disease } \\
\hline Hypertension & $30(68.2)$ & $29(59.2)$ & 0.36 \\
\hline Cerebrovascular disease & $22(50.0)$ & $29(59.2)$ & 0.37 \\
\hline Diabetes & $15(34.1)$ & $17(34.7)$ & 0.95 \\
\hline Cardiovascular disease & $12(27.3)$ & $16(32.7)$ & 0.57 \\
\hline Dementia & $9(20.5)$ & $9(18.4)$ & 0.79 \\
\hline Anxiety/depression & $7(15.9)$ & $12(24.5)$ & 0.30 \\
\hline Chronic lung disease & $6(13.6)$ & $5(10.2)$ & 0.60 \\
\hline Peptic ulcer & $4(9.1)$ & $7(14.3)$ & 0.43 \\
\hline Anemia & $3(6.8)$ & $5(10.2)$ & 0.71 \\
\hline Parkinsonism & $2(4.5)$ & $7(14.3)$ & 0.16 \\
\hline Malignancy except GI origin & $2(4.5)$ & $3(6.1)$ & 0.99 \\
\hline Chronic hepatitis & $2(4.5)$ & $3(6.1)$ & 0.99 \\
\hline Chronic kidney disease & $1(2.3)$ & $3(6.1)$ & 0.61 \\
\hline
\end{tabular}

* Barthel index [26] (0-100) is a scale used to measure performance in basic activities of daily living. A higher number is associated with a better performance. ${ }^{\dagger}$ Participants were classified into three groups according to their bowel performance in the run-in period under the bowel routine protocol in long-term care: group A: received enema more than once a week,

group B: received enema once a week or had less than three times of spontaneous bowel movements per week,

group C: 3-7 times of spontaneous bowel movements per week under usual care.

about whether the study results are reproducible for other research teams or generalizable to the Western patients [29]. By contrast, in our study, the majority of study population in long-term care was estimated to be the pattern of yang deficiency in terms of TCM theories [15]. Therefore, without the need to or help of TCM practitioners, this study is easier to be replicated or translated into routine care of constipation in conventional medicine. However, the relationship of therapeutic effect of $\mathrm{CCH} 1$ with the degree of $y a n g$ deficiency in long stay residents needs to be investigated.
From literature review, there is no consistently applied definition of constipation in the elderly [30]. Definition such as the Rome III criteria, however, may not encompass the perceptions of all patients with constipation [20]. Patients' definitions are often qualitative and include stool consistency, difficulty with passage, requirement of manual, medicinal, or other maneuvers to evacuate feces $[31,32]$. In addition, constipation is often multifactorial in origin (including primary and secondary causes) with mixed types of pathophysiology in long-term care [33]. And the long stay 
TABLE 4: Comparison of the treatment effect between $\mathrm{CCH} 1$ and lactulose.

\begin{tabular}{|c|c|c|c|}
\hline \multirow{2}{*}{ Variables } & \multicolumn{2}{|c|}{ Group, mean $(95 \% \mathrm{CI})$} & \multirow[b]{2}{*}{ Adjusted $P^{\ddagger}$} \\
\hline & $\mathrm{CCH} 1(n=44)$ & Lactulose $(n=49)$ & \\
\hline \multicolumn{4}{|c|}{ Frequency of spontaneous bowel movement $(\mathrm{SBM} / \mathrm{wk})^{\S}$} \\
\hline Baseline (weeks $-2 \sim 0$ ) & $2.7(1.9,3.4)$ & $2.6(2.0,3.2)$ & 0.907 \\
\hline Treatment (weeks 1 4) & $6.9^{\dagger}(6.1,7.6)$ & $4.5^{\dagger}(3.5,5.4)$ & $<0.001$ \\
\hline Treatment (weeks 5 8) & $6.8^{\dagger}(6.0,7.7)$ & $5.5^{\dagger}(4.5,6.6)$ & 0.159 \\
\hline Follow-up (weeks 9 12) & $3.7^{*}(3.1,4.4)$ & $5.2^{\dagger}(4.0,6.4)$ & 0.084 \\
\hline \multicolumn{4}{|c|}{ Frequency of rectal treatment $(\mathrm{RT} / \mathrm{wk})^{\|}$} \\
\hline Baseline (weeks $-2 \sim 0$ ) & $1.4(1.1,1.8)$ & $1.4(1.1,1.7)$ & 0.958 \\
\hline Treatment (weeks 1 4) & $0.5^{\dagger}(0.4,0.7)$ & $0.9^{\dagger}(0.7,1.2)$ & 0.030 \\
\hline Treatment (weeks 5 8) & $0.3^{\dagger}(0.2,0.5)$ & $0.6^{\dagger}(0.4,0.8)$ & 0.051 \\
\hline Followup (weeks 9 12) & $1.0^{*}(0.8,1.3)$ & $0.8^{\dagger}(0.5,1.1)$ & 0.561 \\
\hline \multicolumn{4}{|c|}{ Amount of rescue laxative use $(\mathrm{MgO} / \mathrm{wk})$} \\
\hline Baseline (weeks $-2 \sim 0$ ) & $0(0,0)$ & $0.4(-0.1,0.8)$ & 0.083 \\
\hline Treatment (weeks 1 4) & $0.2(-0.1,0.5)$ & $2.4^{*}(1.1,3.8)$ & 0.005 \\
\hline Treatment (weeks 5 8) & $1.7(-0.4,3.9)$ & $10.4^{\dagger}(6.1,14.7)$ & 0.002 \\
\hline Followup (weeks 9 12) & $19.1^{\dagger}(14.9,23.2)$ & $20.0^{\dagger}(14.5,25.4)$ & 1.000 \\
\hline \multicolumn{4}{|l|}{ Laxative cost (USD/wk) } \\
\hline Treatment (weeks 1 4) & $1.4(1.2,1.5)$ & $4.7(4.2,5.2)$ & $<0.001$ \\
\hline Treatment (weeks 5 8) & $1.5(1.3,1.7)$ & $5.0(4.4,5.7)$ & $<0.001$ \\
\hline
\end{tabular}

Pairwise comparisons were performed for each treatment group with its baseline.

$* P<0.01 ;{ }^{\dagger} P<0.001$.

$¥ P$ value was adjusted by Bonferroni adjustment.

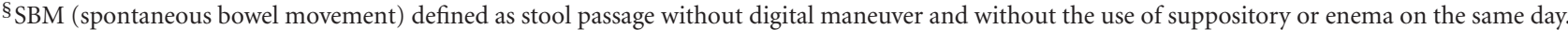

"RT (rectal treatment) including enema, suppository use, or digital maneuver.

"Laxative cost including the acquisition costs of study medication and rescue laxative.

residents in our study had potentially poor cognitive function or expression ability. Therefore, we added the other two inclusion criteria of constipation ("receiving enema, suppository or digital maneuver" and "laxative use") to Rome III criteria, which are composed of several "subjective" complaints for "functional" constipation.

For the classification of constipation severity, the residents who had bowel frequency of 3-7 times a week during run-in period were referred to as group C (mild constipation). Although physicians often use the quantitative definition of $<3$ bowel movements per week to describe constipation, there is lack of agreement on the definition of constipation regarding what patients perceive and what physicians traditionally see as constipation [1]. Furthermore, placebo was given in the run-in period, which is associated with high rates of resolution in a functional bowel disorder [34]. And variation in symptoms over time is the nature of functional disorders, which may contribute to the "temporary" improvement of constipation during run-in period. Therefore, group $\mathrm{C}$ patients are not excluded as the study design of a previous clinical trial [17].

Moreover, since high percentage of residents in longterm care had precipitants of constipation including medical conditions and related medications [3], exclusion of these patients in clinical trial of constipation in long stay is neither practical nor ethical. Therefore, we kept the patients and investigated their medical illness. After randomization, major diseases in the patients were equally distributed between both groups as described in Table 3 . In addition, for ethical reasons, all medications except laxatives were left for participants as prescribed before enrolled. Although there was no detailed record or analysis about every category of medication that patients used during the study, it is reasonable to infer that disease-related medications were randomly distributed and balanced between the two groups as that of medical illness. However, further study with explicit survey of related medications is still essential.

For the primary outcome of SBMs, the mean numbers of weekly SBM in CCH1 and lactulose groups were $6.8 \pm 2.2$ and $5.0 \pm 2.9$, respectively, during 8 -week treatment phase. The pooled standard deviation was calculated to be 2.6 , which is slightly larger than the assumed one $(\mathrm{SD}=2.0)$ used in the sample size calculation. However, the observed effect size here (1.8) is also larger than the expected value of 1.5 bowel movements, making the standardized effect size of 0.7 $(1.8 / 2.6)$ close to the expected one of $0.75(1.5 / 2.0)$. Therefore, after calculation by the software package for sample size and power estimation PASS (Power Analysis and Sample Size, Kaysville, UT, USA), we still have the power of 0.91 to tell the difference of SBM frequency between the two groups. With regard to the negative results of secondary outcomes, although it could be attributed to the lower power $(0.1-0.5)$ to identify the differences between groups, some of these effect sizes observed here were actually small and clinically 
TABLE 5: Comparison of stool consistency and stool amount between CCH1 $(n=44)$ and lactulose $(n=49)$ groups.

\begin{tabular}{|c|c|c|c|}
\hline \multirow{2}{*}{ Variables } & \multicolumn{2}{|c|}{ Group, mean $(95 \% \mathrm{CI})$} & \multirow[b]{2}{*}{ Adjusted $P^{*}$} \\
\hline & $\mathrm{CCH} 1$ & Lactulose & \\
\hline \multicolumn{4}{|c|}{ Average score of bristol scale ${ }^{\dagger}$} \\
\hline Week $-2-0$ & $4.0(3.8,4.3)$ & $4.2(4.0,4.3)$ & 0.495 \\
\hline Week 1-4 & $4.7(4.5,4.9)$ & $4.5(4.3,4.7)$ & 0.475 \\
\hline Week 5-8 & $4.7(4.5,4.9)$ & $4.8(4.6,5.0)$ & 1.000 \\
\hline Week 9-12 & $4.5(4.3,4.8)$ & $4.7(4.5,4.9)$ & 1.000 \\
\hline \multicolumn{4}{|c|}{ Percentage of large stool amount ${ }^{\ddagger}$} \\
\hline Week $-2-0$ & $29.8(21.5,38.1)$ & $32.9(25.4,40.3)$ & 0.581 \\
\hline Week 1-4 & $36.6(30.5,42.8)$ & $38.3(30.8,45.9)$ & 1.000 \\
\hline Week 5-8 & $42.1(34.9,49.2)$ & $38.7(31.4,46.0)$ & 1.000 \\
\hline Week 9-12 & $37.0(29.8,44.3)$ & $32.9(25.2,40.6)$ & 1.000 \\
\hline \multicolumn{4}{|c|}{ Percentage of moderate stool amount ${ }^{\ddagger}$} \\
\hline Week $-2-0$ & $50.5(42.0,59.1)$ & $52.9(44.5,61.2)$ & 0.692 \\
\hline Week 1-4 & $52.2(47.1,57.4)$ & $49.2(41.0,57.4)$ & 1.000 \\
\hline Week 5-8 & $48.2(41.9,54.6)$ & $47.5(39.9,55.0)$ & 1.000 \\
\hline Week 9-12 & $53.2(46.0,60.4)$ & $50.9(42.9,58.9)$ & 1.000 \\
\hline \multicolumn{4}{|c|}{ Percentage of small stool amount ${ }^{\ddagger}$} \\
\hline Week $-2-0$ & $19.7(13.1,26.3)$ & $14.2(8.6,19.9)$ & 0.211 \\
\hline Week 1-4 & $11.1(7.4,14.8)$ & $12.0(7.5,16.4)$ & 1.000 \\
\hline Week 5-8 & $9.7(5.6,13.8)$ & $13.8(8.9,18.7)$ & 0.588 \\
\hline Week 9-12 & $9.8(6.1,13.5)$ & $16.2(11.0,21.4)$ & 0.142 \\
\hline
\end{tabular}

${ }^{*} P$ value was adjusted by Bonferroni adjustment.

${ }^{\dagger}$ Stool consistency was recorded by Bristol Stool Form Scale [25], ranging from 1 (separate hard lumps, like nuts) to 7 (watery, no solid pieces).

${ }^{\ddagger}$ Stool amount was classified into three categories (small, $<250 \mathrm{~g}$; moderate, 250-500 g; large, $>500 \mathrm{~g}$ ) according to a national guidelines of the Registered Nurses Association [24].

TABLE 6: Global assessment of efficacy. ${ }^{*, \dagger}$

\begin{tabular}{lccc}
\hline \multirow{2}{*}{ Variables } & \multicolumn{2}{c}{ Group, number $(\%)$} & \multirow{2}{*}{ C Value } \\
& CCH1 & Lactulose & \\
\hline Marked improved & $21(53.8)$ & $18(52.9)$ & \\
Slightly improved & $12(30.8)$ & $9(26.5)$ & \\
Unchanged & $5(12.8)$ & $7(20.6)$ & 0.646 \\
Slightly worse & $1(2.6)$ & $0(0)$ & \\
Markedly worse & $0(0)$ & $0(0)$ & \\
\hline
\end{tabular}

${ }^{*}$ The global assessment of efficacy was evaluated at the end of treatment phase by patients themselves or their principal caregivers if their cognition was impaired.

${ }^{\dagger}$ A total of 20 patients dropped out of the trial during the treatment phase.

insignificant. Further study with larger sample size may be needed to identify the significance of the outcome measures with small effect size.

Although the prespecified LOCF was applied as our analytic method, it could not be the most appropriate way to deal with the missing data. It is of particular concern that the dropout rate was different between the two groups during study period. Because more dropouts in the lactulose group came from "adverse events" and all of them were hospitalized for "severe adverse events", it is reasonable to infer that these dropout patients' constipation would become worse after
TABLe 7: Compliance for study medication.*

\begin{tabular}{lccc}
\hline \multirow{2}{*}{ Study medication } & \multicolumn{2}{c}{ Group, mean (SD) } & P Value \\
& CCH1 & Lactulose & \\
\hline $\begin{array}{l}\text { Powder } \\
\text { CCH1 }\end{array}$ & $97.1(6.4)$ & - & 0.772 \\
CCH1 Placebo & - & $97.4(5.1)$ & \\
Syrup & & & \\
Lactulose & - & $97.5(5.1)$ & 0.637 \\
$\quad$ Lactulose Placebo & $96.9(6.4)$ & - & \\
* The experimental group received CCH1 powder and lactulose placebo. The \\
active comparator group was given lactulose syrup and CCH1 placebo.
\end{tabular}

TABLE 8: Comparison of severe adverse events.

\begin{tabular}{lcc}
\hline \multirow{2}{*}{ Variables } & \multicolumn{2}{c}{ Group, number (\%) } \\
& CCH1 & Lactulose \\
\hline Pneumonia & $5(11.4)$ & $5(10.2)$ \\
Upper GI bleeding & $1(2.3)$ & $1(2.0)$ \\
Acute gastroenteritis & $0(0)$ & $2(4.1)$ \\
Cellulitis & $0(0)$ & $1(2.0)$ \\
Urinary tract infection & $0(0)$ & $2(4.1)$ \\
Ileus & $0(0)$ & $1(2.0)$ \\
\hline
\end{tabular}


TABLE 9: Comparison of common adverse events (cumulative incidence $>5 \%$ ).

\begin{tabular}{|c|c|c|c|}
\hline \multirow{2}{*}{ Variables } & \multicolumn{2}{|c|}{ Group, number (\%) } & \multirow{2}{*}{$P$ Value } \\
\hline & $\mathrm{CCH} 1$ & Lactulose & \\
\hline Flatulence & $16(36.4)$ & $17(34.7)$ & 0.867 \\
\hline Albumin $\downarrow$ & $15(34.1)$ & $10(20.4)$ & 0.137 \\
\hline Diarrhea & $15(34.1)$ & $17(34.7)$ & 0.951 \\
\hline Hiccup & $13(29.5)$ & $12(24.5)$ & 0.583 \\
\hline Hemoglobin $\downarrow$ & $12(27.3)$ & $6(12.2)$ & 0.067 \\
\hline Abdominal pain & $10(22.7)$ & $8(16.3)$ & 0.435 \\
\hline Bloating & $9(20.5)$ & $11(22.4)$ & 0.815 \\
\hline Magnesium $\uparrow$ & $8(18.2)$ & $12(24.5)$ & 0.614 \\
\hline Acid regurgitation & $8(18.2)$ & $8(16.3)$ & 0.813 \\
\hline URI & $8(18.2)$ & $6(12.2)$ & 0.424 \\
\hline Red blood cell $\downarrow$ & $7(15.9)$ & $4(8.2)$ & 0.248 \\
\hline Sugar $\uparrow$ & $6(13.6)$ & $9(18.4)$ & 0.536 \\
\hline Calcium $\downarrow$ & $6(13.6)$ & $2(4.1)$ & 0.143 \\
\hline Sodium $\downarrow$ & $5(11.4)$ & $2(4.1)$ & 0.249 \\
\hline Alkaline phosphatase $\uparrow$ & $4(9.1)$ & $1(2.0)$ & 0.186 \\
\hline Albumin $\uparrow$ & $4(9.1)$ & $1(2.0)$ & 0.186 \\
\hline Potassium $\uparrow$ & $4(9.1)$ & $4(8.2)$ & 1.000 \\
\hline Phosphate $\uparrow$ & $4(9.1)$ & $1(2.0)$ & 0.186 \\
\hline Nausea & $4(9.1)$ & $7(14.3)$ & 0.439 \\
\hline White blood cell $\downarrow$ & $3(6.8)$ & $2(4.1)$ & 0.665 \\
\hline Sugar $\downarrow$ & $3(6.8)$ & $2(4.1)$ & 0.665 \\
\hline Blood urea nitrogen $\uparrow$ & $3(6.8)$ & $5(10.2)$ & 0.718 \\
\hline Dizziness & $3(6.8)$ & $1(2.0)$ & 0.341 \\
\hline Cough & $3(6.8)$ & $4(8.2)$ & 0.561 \\
\hline Skin rash & $2(4.5)$ & $3(6.1)$ & 1.000 \\
\hline Platelet $\uparrow$ & $2(4.5)$ & $3(6.1)$ & 1.000 \\
\hline Aspartate aminotransferase $\uparrow$ & $2(4.5)$ & $4(8.2)$ & 0.680 \\
\hline Alanine aminotransferase $\uparrow$ & $2(4.5)$ & $3(6.1)$ & 1.000 \\
\hline Creatinine $\uparrow$ & $2(4.5)$ & $3(6.1)$ & 1.000 \\
\hline Triglyceride $\uparrow$ & $2(4.5)$ & $5(10.2)$ & 0.440 \\
\hline Vomiting & $2(4.5)$ & $3(6.1)$ & 1.000 \\
\hline Diaper rash & $1(2.3)$ & $5(10.2)$ & 0.207 \\
\hline Cholesterol $\uparrow$ & $1(2.3)$ & $4(8.2)$ & 0.365 \\
\hline Cholesterol $\downarrow$ & $1(2.3)$ & $3(6.1)$ & 0.619 \\
\hline Creatine phosphokinase $\uparrow$ & $0(0)$ & $3(6.1)$ & 0.244 \\
\hline Uric acid $\uparrow$ & $0(0)$ & $3(6.1)$ & 0.244 \\
\hline Pruritus & $0(0)$ & $3(6.1)$ & 0.244 \\
\hline Chest tightness & $0(0)$ & $3(6.1)$ & 0.244 \\
\hline
\end{tabular}

${ }^{*} \mathrm{~A} \chi^{2}$ test or Fisher exact test where appropriate was used to compare the number of patients with adverse events between two groups.

admission. Our estimation in the outcome variables at endpoint for the lactulose group would result in a better status under the imputation method of LOCF because of its assumption that the response remains constant at the last observed value. Under this condition, the efficacy of $\mathrm{CCH} 1$ group would be underestimated. Thus, the biased results in the effect are toward the null, a lesser threat to validity. In addition, LOCF was the most common method that was used in the literature [35], and mixed models for handling the missing data do not work well due to our small size of sample [36].

According to a recent systematic review [18] of 137 studies on the efficacy of TCM for the management of constipation, only 21 clinical trials were high of quality with participants' mean age of 50 years and TCM intervention of 20.6 days. Compared with them, our study has older participants of 73 years and longer intervention of 2 months. Among the 21 clinical trials of high quality, there were eight studies to 
compare TCM with Western medicine, which were all published in Chinese, and the results showed that Chinese herbal medicine were more effective than conventional medicines (cisapride, mosapride, or phenolphthalein). Compared with all the active controls selected in the eight studies, only lactulose used in our study had good supporting evidence for the treatment of chronic constipation $[19,37]$. Therefore, $\mathrm{CCH} 1$ is the first Chinese herbal Medicine, which results of this study suggesting a better efficacy than lactulose as a treatment option for constipation in long-term care.

The double-blind, randomized, and placebo-controlled trial is the gold standard method to test the efficacy of a new treatment [38]. However, the herbal supplements with quality placebo-controlled trials to evaluate their effect on constipation were limited from the literature review $[18,19]$. Furthermore, for the long-term care setting, little evidence is available from high-quality randomized controlled trials [39]. The tincture of jalapa, derived from a tropical plant in Brazil, is shown to be effective in the acute treatment of functional constipation [40]. Yun-chang capsule, a Chinese herbal formula, is efficacious and safe on the treatment of functional constipation for patients with deficient syndrome of $Q i$ and yin [41]. Hemp Seed Pill, a TCM proprietary medicine, is safe and effective for alleviating functional constipation for subjects in excessive syndrome [35]. The study population in the three herbal trials was all targeted to the ambulatory outpatients with mean age of 33-42 years [35, $40,41]$. For the residents in long-term care, Smooth Move herbal tea, when added to the standard treatment with usual laxatives, increased bowel movements compared to the addition of a placebo tea [42]. In comparison with Smooth Move as a complementary agent for current laxatives, $\mathrm{CCH} 1$ in our study was documented to be a good alternative laxative to lactulose as first-line treatment for long stay patients with constipation.

Compared with lactulose used in our study, although polyethylene glycol (PEG) has not been studied in trials of exclusively geriatric populations [43], a Cochrane review indicates that PEG is better than lactulose in outcomes of stool frequency and stool consistency [44]. However, a drug class review conducted by the FDA noted that high doses of PEG may produce diarrhea and excessive stool frequency, particularly in elderly nursing home patients [45]. Lactulose and the other nonabsorbable sugar, sorbitol, appeared to be equally efficacious, but sorbitol is recommended as a cost-effective alternative to lactulose for the treatment of constipation in the elderly [46]. Lactitol, a widely prescribed osmotic laxative in India, is also suggested to be preferred over lactulose for adults with chronic constipation because of its comparable efficacy, better palatability and lesser incidence of adverse events [47]. In addition, a combination of senna plus fibre is more cost-effective and efficient than lactulose in treating constipation in long stay elderly patients [48]. Therefore, further studies to compare the efficacy, safety, and cost-effectiveness of CCH1 with other osmotic agents, including PEG, sorbitol, lactitol, or combination laxatives for the treatment of constipation in long-term care are encouraged.
There are some strengths in our study. The clinical trial of head-to-head comparison of $\mathrm{CCH} 1$ and lactulose was conducted with a strict and accepted methodological protocol. Compliance was around $97 \%$, which is excellent for a trial in older subjects. Furthermore, unlike most randomized controlled trials for constipation conducted in ideal conditions [39] or in the population of ambulatory communitydwelling adults $[19,49]$, this study was conducted in the real situation without interference with the usual care and bowel routine for the long-term care residents, who have many nonmodifiable risk factors such as polypharmacy and coexistent medical conditions. In addition, the clinical trial fully adheres to the rationale of medical prescription in conventional medicine, which usually prescribing initial dosage of laxatives based on the severity of constipation, then titrating according to drug response.

This study has some limitations or weakness. First, test of success of blinding was not performed in the study. Second, because the residents in long-term care had potentially impaired cognitive function or poor expression ability due to old age with frailty, multiple medication, and chronic illness, objective data from medical records and stool diaries were used for outcome measurements rather than the subjective assessment of symptom score or quality of life. Third, the main population in the study was older adults living in longterm care. Therefore, the effect of CCH1 for young residents in long stay or the elderly in the community remains to be investigated. Fourth, the medical cost in the study was limited to the pharmacological cost of national health insurance in Taiwan, which is not generalizable to different countries or different health insurance systems. Moreover, the true cost of managing constipation is impacted on by a broad range of resources and not only laxative acquisition costs [50]. Therefore, further clinical trials may be necessary to compare the economic impact of CCH1 with current laxatives, including time, health providers' visits, caregivers' burden, and labor cost. Fifth, for the considerations of ethical concerns and real situation in clinical practice, when study medication was titrated to maximal dose, our study design allowed the primary physician to add and adjust the dosage of rescue laxative every week until optimal bowel performance was observed. Therefore, the laxative effect in the study was probably attributed to the combined effect of study medication and $\mathrm{MgO}$. In addition, because $\mathrm{CCH} 1$ had better laxative effect than lactulose on SBM during treatment, the weekly amount of $\mathrm{MgO}$ was less in $\mathrm{CCH} 1$ group than that used in the lactulose group during treatment phase and the beginning of follow-up phase (4.2 versus 14.1 at week 9, $P=0.002$ ). Therefore, we did not have enough evidence to compare the maintenance effect of treatment medication by the study design.

\section{Conclusions}

In conclusion, both $\mathrm{CCH} 1$ and lactulose were efficacious and well tolerated but $\mathrm{CCH} 1$ significantly increased spontaneous bowel movements and concurrently reduced the need of rectal treatments and additional laxative at lower laxative cost during treatment. Although the maintenance effect of CCH1 
was uncertain, it still suggested good value for new drug development. A properly designed large-scale or multicenter clinical trial with longitudinal dada analysis is essential. Further comparative study with other laxatives is also encouraged, particularly with regard to concerns about quality of life and subjective outcomes.

\section{Funding}

The study sponsor did not participate in the study design and conduct; collection, management, analysis, and interpretation of the data; preparation, review, or approval of the paper.

\section{Conflict of Interests}

The authors declare that they have no conflict of interests.

\section{Acknowledgment}

This study was supported by the research fund from National Taiwan University Hospital Yun-Lin Branch (NTUHYL 97.N0023).

\section{References}

[1] S. H. Tariq, "Constipation in long-term care," Journal of the American Medical Directors Association, vol. 8, no. 4, pp. 209218, 2007.

[2] D. Harari, J. H. Gurwitz, J. Avorn, R. Bohn, and K. L. Minaker, "Bowel habit in relation to age and gender. Findings from the national health interview survey and clinical implications," Archives of Internal Medicine, vol. 156, no. 3, pp. 315-320, 1996.

[3] C. Phillips, D. Polakoff, S. K. Maue, and R. Mauch, "Assessment of constipation management in long-term care patients," Journal of the American Medical Directors Association, vol. 2, no. 4, pp. 149-154, 2001.

[4] E. P. Bouras and E. G. Tangalos, "Chronic constipation in the elderly," Gastroenterology Clinics of North America, vol. 38, no. 3, pp. 463-480, 2009.

[5] C. Dennison, M. Prasad, A. Lloyd, S. K. Bhattacharyya, R. Dhawan, and K. Coyne, "The health-related quality of life and economic burden of constipation," PharmacoEconomics, vol. 23, no. 5, pp. 461-476, 2005.

[6] L. Frank, J. Schmier, L. Kleinman et al., "Time and economic cost of constipation care in nursing homes," Journal of the American Medical Directors Association, vol. 3, no. 4, pp. 215223, 2002.

[7] H. A. Tindle, R. B. Davis, R. S. Phillips, and D. M. Eisenberg, "Trends in use of complementary and alternative medicine by US adults: 1997-2002," Alternative Therapies in Health and Medicine, vol. 11, no. 1, pp. 42-49, 2005.

[8] C. L. Lu, "Chinese herbal medicine for constipation in Taiwan," Journal of the Chinese Medical Association, vol. 73, no. 10, pp. 511-512, 2010.

[9] M. S. Jong, S. J. , Hwang, Y. C. Chen, T. J. Chen, F. J. Chen, and F. P. Chen, "Prescriptions of Chinese herbal medicine for constipation under the national health insurance in Taiwan," Journal of the Chinese Medical Association, vol. 73, no. 7, pp. 375-383, 2010.

[10] L. C. Chen, B. R. Wang, Y. C. Chou, and J. H. Tien, "Drug utilization pattern of Chinese herbal medicines in a general hospital in Taiwan," Pharmacoepidemiology and Drug Safety, vol. 14, no. 9, pp. 651-657, 2005.

[11] G. Maciocia, The Foundations of Chinese Medicine: A Comprehensive Text for Acupuncturists and Herbalists, Churchill Livingstone, London, UK, 2nd edition, 2005.

[12] W. Maclean and J. Lyttleton, Clinical Handbook of Internal Medicine: The Treatment of Disease with Traditional Chinese Medicine, University of Western Sydney, Syndney, Australia, 2002.

[13] WHO International Standard Terminologies on Tradtional Medicine in the Western Pacific Region, Publications of the World Health Organization, Geneva, Switzerland, 2007.

[14] X. C. Xu, "Purgative prescriptions composed of drugs warm in nature," in Pharmacology of Traditional Chinese Medical Formulae, X. C. Xu, Ed., p. 57, Higher Education Press, Beijing, China, 1994.

[15] C. Liu, A. Tseng, and S. Yang, Chinese Herbal Medicine: Modern Applications of Traditional Formulas, CRC Press LLC, New York, NY, USA, 2005.

[16] X. C. Xu, “The prescription of Wenpi Tang," in Pharmacology of Traditional Chinese Medical Formulae, X. C. Xu, Ed., pp. 5961, Higher Education Press, Beijing, China, 1994.

[17] C. H. Huang, Y. C. Su, T. C. Li et al., "Treatment of constipation in long-term care with Chinese herbal formula: a randomized, double-blind placebo-controlled trial," Journal of Alternative and Complementary Medicine, vol. 17, no. 7, pp. 639-646, 2011.

[18] L. W. Lin, Y. T. Fu, T. Dunning et al., "Efficacy of traditional Chinese medicine for the management of constipation: a systematic review," Journal of Alternative and Complementary Medicine, vol. 15, no. 12, pp. 1335-1346, 2009.

[19] L. J. Brandt, C. M. Prather, E. M. Quigley, L. R. Schiller, P. Schoenfeld, and N. J. Talley, "Systematic review on the management of chronic constipation in North America," The American Journal of Gastroenterology, vol. 100, supplement 1, pp. S5-S21, 2005.

[20] V. Fleming and W. E. Wade, "A review of laxative therapies for treatment of chronic constipation in older adults," The American Journal of Geriatric Pharmacotherapy, vol. 8, no. 6, pp. 514-550, 2010.

[21] M. Petticrew, I. Watt, and T. Sheldon, "Systematic review of the effectiveness of laxatives in the elderly," Health Technology Assessment, vol. 1, no. 13, pp. 1-52, 1997.

[22] K. Kelly-Pieper, S. P. Patil, P. Busse et al., "Safety and tolerability of an antiasthma herbal Formula (ASHMI) in adult subjects with asthma: a randomized, double-blinded, placebocontrolled, dose-escalation phase I study," Journal of Alternative and Complementary Medicine, vol. 15, no. 7, pp. 735-743, 2009.

[23] J. F. Sanders, "Lactulose syrup assessed in a double-blind study of elderly constipated patients," Journal of the American Geriatrics Society, vol. 26, no. 5, pp. 236-239, 1978.

[24] Prevention of Constipation in the Older Adult Population, Registered Nurses Association of Ontario. National Guideline Clearinghouse, 2005.

[25] S. J. Lewis and K. W. Heaton, "Stool form scale as a useful guide to intestinal transit time," Scandinavian Journal of Gastroenterology, vol. 32, no. 9, pp. 920-924, 1997.

[26] F. I. Mahoney and D. W. Barthel, "Functional evaluation: the barthel index," Maryland State Medical Journal, vol. 14, pp. 6165, 1965.

[27] K. S. Dua, R. Shaker, T. R. Koch, and J. S. Bajaj, "Gastroenterologic disorders," in Practice of Geriatrics, E. H. Duthie, P. R. 
Katz, and M. L. Malone, Eds., pp. 592-596, Saunders Elsevier, Philadelphia, Pa, USA, 4th edition, 2007.

[28] Y. C. Su, L. L. Chen, J. D. Lin, J. S. Lin, Y. C. Huang, and J. S. Lai, "BCQ+: a body constitution questionnaire to assess Yang-Xu. Part I: establishment of a first final version through a Delphi process," Forschende Komplementärmedizin, vol. 15, no. 6, pp. 327-334, 2008.

[29] R. L. Whiting and A. C. Ford, "Efficacy of traditional Chinese medicine in functional constipation," The American Journal of Gastroenterology, vol. 106, no. 5, p. 1003, 2011.

[30] D. Harari, J. H. Gurwitz, and K. L. Minaker, "Constipation in the elderly," Journal of the American Geriatrics Society, vol. 41, no. 10, pp. 1130-1140, 1993.

[31] D. Harari, J. H. Gurwitz, J. Avorn, R. Bohn, and K. L. Minaker, "How do older persons define constipation? Implications for therapeutic management," Journal of General Internal Medicine, vol. 12, no. 1, pp. 63-66, 1997.

[32] P. Pare, S. Ferrazzi, W. G. Thompson, E. J. Irvine, and L. Rance, "An epidemiological survey of constipation in Canada: definitions, rates, demographics, and predictors of health care seeking," The American Journal of Gastroenterology, vol. 96, no. 11, pp. 3130-3137, 2001.

[33] S. A. Dosh, "Evaluation and treatment of constipation," The Journal of Family Practice, vol. 51, no. 6, pp. 555-559, 2002.

[34] P. Enck and S. Klosterhalfen, "The placebo response in functional bowel disorders: perspectives and putative mechanisms," Neurogastroenterology and Motility, vol. 17, no. 3, pp. 325-331, 2005.

[35] C. W. Cheng, Z. X. Bian, L. X. Zhu, J. C. Wu, and J. J. Sung, "Efficacy of a Chinese herbal proprietary medicine (Hemp Seed Pill) for functional constipation," The American Journal of Gastroenterology, vol. 106, no. 1, pp. 120-129, 2011.

[36] R. M. Hamer and P. M. Simpson, "Last observation carried forward versus mixed models in the analysis of psychiatric clinical trials," The American Journal of Psychiatry, vol. 166, no. 6, pp. 639-641, 2009.

[37] C. W. Cheng, Z. X. Bian, and T. X. Wu, "Systematic review of Chinese herbal medicine for functional constipation," World Journal of Gastroenterology, vol. 15, no. 39, pp. 4886-4895, 2009.

[38] E. J. Irvine, W. E. Whitehead, W. D. Chey et al., "Design of treatment trials for functional gastrointestinal disorders," Gastroenterology, vol. 130, no. 5, pp. 1538-1551, 2006.

[39] H. Y. Cheng, "Evidence-based medicine (EBM): what longterm care providers need to know," Clinics in Geriatric Medicine, vol. 27, no. 2, pp. 193-198, 2011.

[40] G. H. Cunha, F. V. Fechine, L. K. Santos et al., "Efficacy of the tincture of jalapa in the treatment of functional constipation: a double-blind, randomized, placebo-controlled study," Contemporary Clinical Trials, vol. 32, no. 2, pp. 153-159, 2011.

[41] G. Jia, M. B. Meng, Z. W. Huang et al., "Treatment of functional constipation with the Yun-chang capsule: a doubleblind, randomized, placebo-controlled, dose-escalation trial," Journal of Gastroenterology and Hepatology, vol. 25, no. 3, pp. 487-493, 2010.

[42] S. Bub, J. Brinckmann, G. Cicconetti, and B. Valentine, "Efficacy of an herbal dietary supplement (Smooth Move) in the management of constipation in nursing home residents: a randomized, double-blind, placebo-controlled study," Journal of the American Medical Directors Association, vol. 7, no. 9, pp. 556-561, 2006.

[43] V. B. Kalish, B. Loven, and M. Sehgal, "Clinical inquiries. What is the best treatment for chronic constipation in the elderly,"
The Journal of Family Practice, vol. 56, no. 12, pp. 1050-1052, 2007.

[44] H. Lee-Robichaud, K. Thomas, J. Morgan, and R. L. Nelson, "Lactulose versus polyethylene glycol for chronic constipation," The Cochrane Database of Systematic Reviews, no. 7, 2010, CD007570.

[45] G. Garlehner, D. E. Jonas, L. C. Morgan et al., Drug Class Review on Constipation Drugs, Oregon Health \& Science University, Portland, Ore, USA, 2007.

[46] F. A. Lederle, D. L. Busch, K. M. Mattox, M. J. West, and D. M. Aske, "Cost-effective treatment of constipation in the elderly: a randomized double-blind comparison of sorbitol and lactulose," The American Journal of Medicine, vol. 89, no. 5, pp. 597-601, 1990.

[47] "Lactitol or lactulose in the treatment of chronic constipation: result of a systematic," Journal of the Indian Medical Association, vol. 108, no. 11, pp. 789-792, 2010.

[48] A. P. Passmore, K. Wilson-Davies, C. Stoker, and M. E. Scott, "Chronic constipation in long stay elderly patients: a comparison of lactulose and a senna-fibre combination," British Medical Journal, vol. 307, no. 6907, pp. 769-771, 1993.

[49] D. Ramkumar and S. S. Rao, "Efficacy and safety of traditional medical therapies for chronic constipation: systematic review," The American Journal of Gastroenterology, vol. 100, no. 4, pp. 936-971, 2005.

[50] A. H. Christie, P. Culbert, and J. F. Guest, "Economic impact of low dose polyethylene glycol 3350 plus electrolytes compared with lactulose in the management of idiopathic constipation in the UK," PharmacoEconomics, vol. 20, no. 1, pp. 49-60, 2002. 


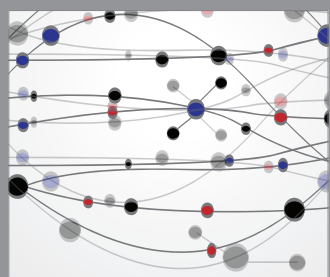

The Scientific World Journal
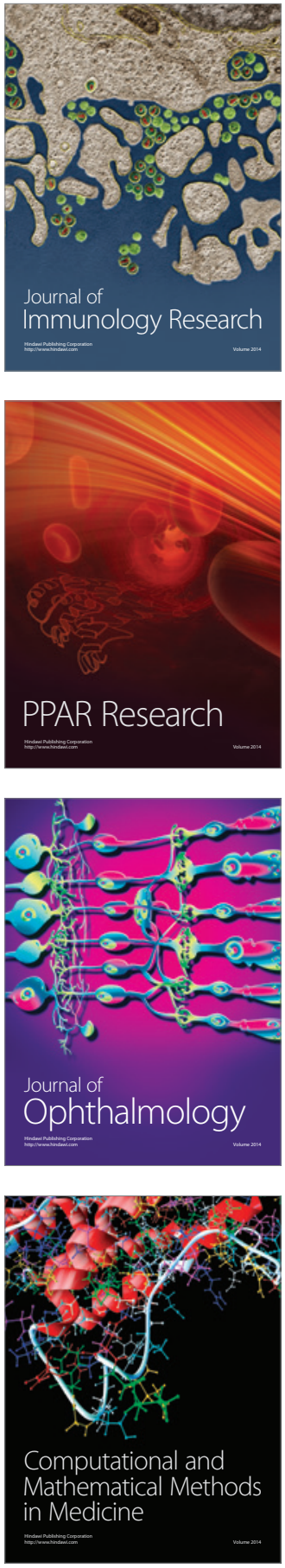

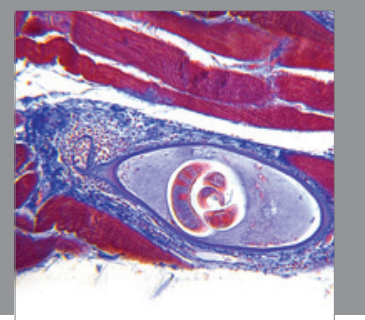

Gastroenterology

Research and Practice
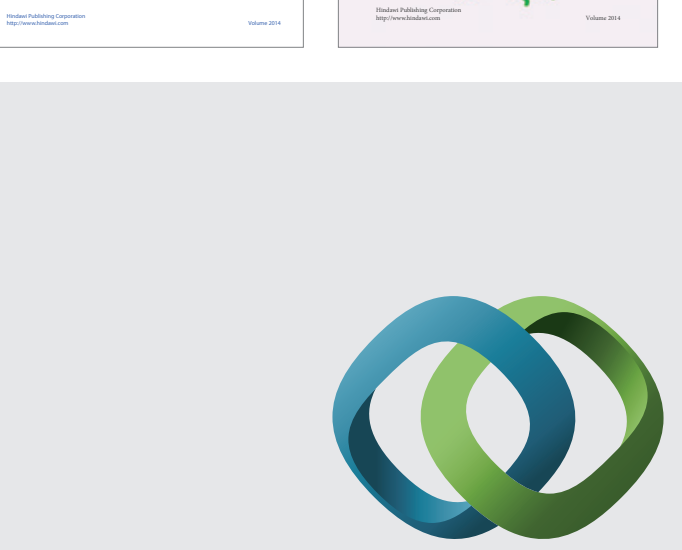

\section{Hindawi}

Submit your manuscripts at

http://www.hindawi.com
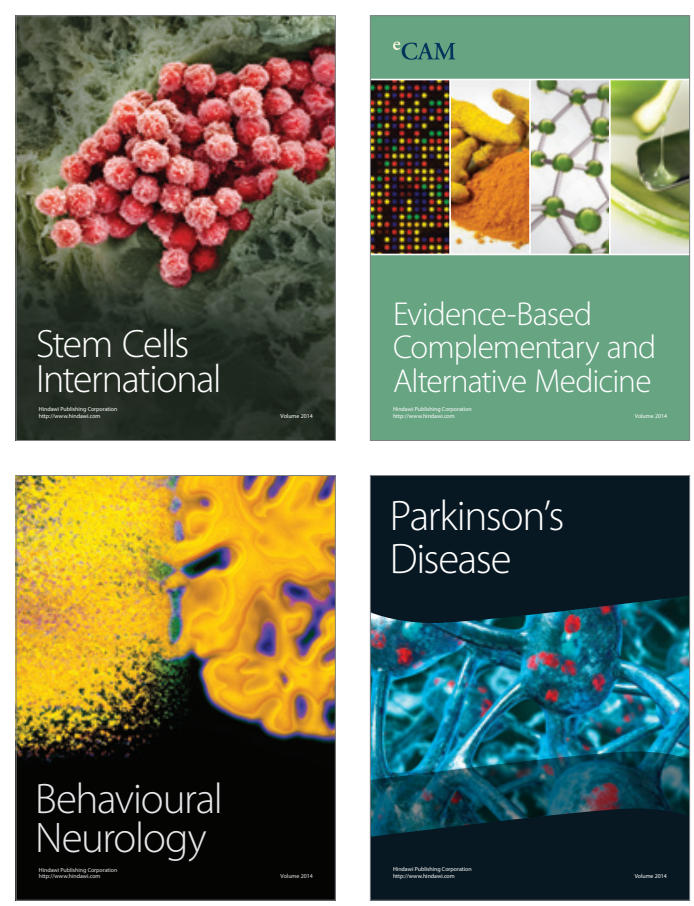

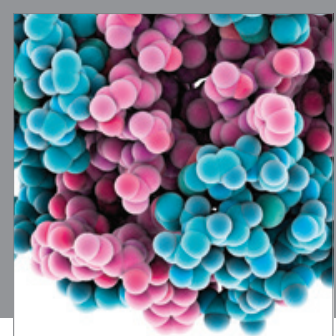

Journal of
Diabetes Research

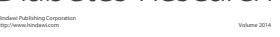

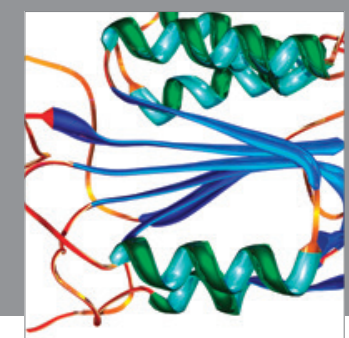

Disease Markers
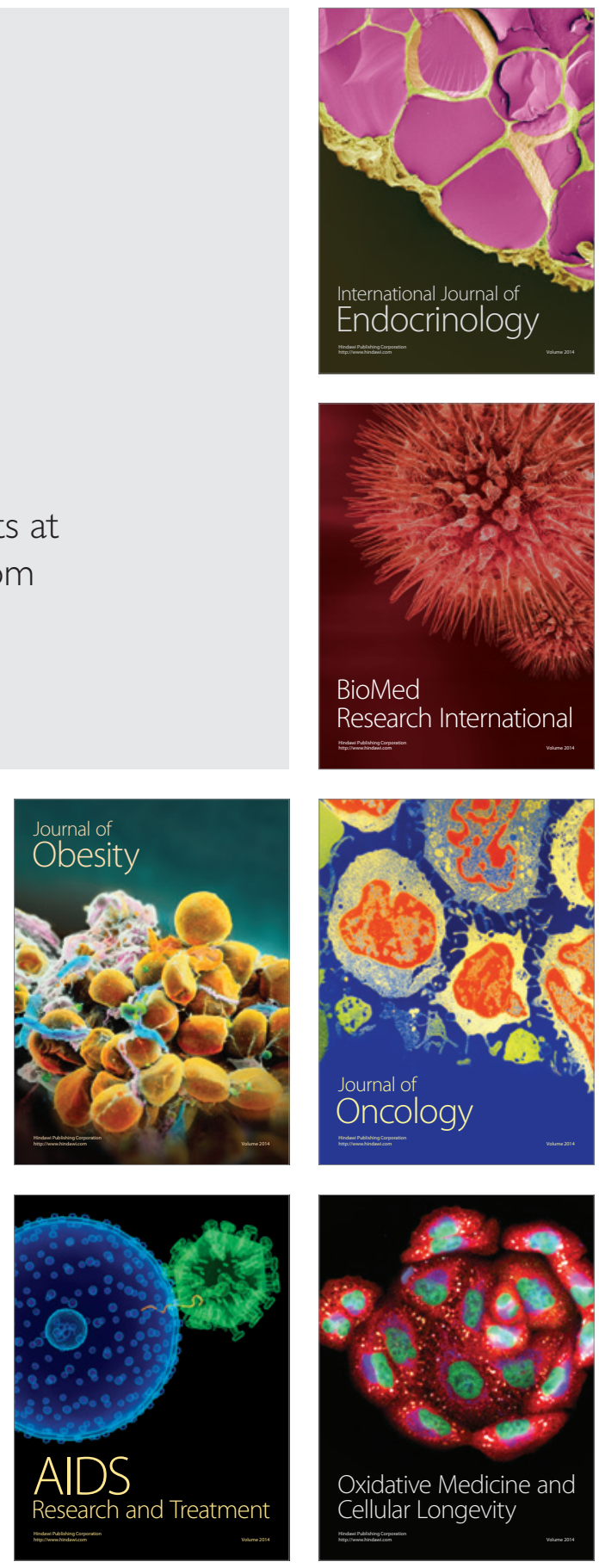